\title{
Editorial
}

\section{Los Recursos Humanos en el sector de la Prevención. Soluciones Formativas}

La publicación en 1997 del Reglamento de los Servicios de Prevención configuró legalmente una estructura profesional donde se establecía que cada Servicio de Prevención acreditado para el desarroLlo de la disciplina preventiva denominada "Medicina del Trabajo" y, en concreto, para el ejercicio de las funciones de nivel superior previstas en el artículo 37, Apdo. 3, conocidas, genéricamente como la "Vigilancia de la Salud a Riesgos específicos", debería incorporar AL MENOS 1 médico y 1 enfermero con titulación específica.

Esas titulaciones específicas incluían varias denominaciones según el propio Decreto, en el caso del médico: Médicos Especialista en Medicina del Trabajo y Diplomados en Medicina de Empresa y, en el caso de la enfermera, ATS de Empresa y Diplomados en Enfermería de Empresa

Esta diversidad de titulaciones aludía a títulos y diplomas que ya existian a la fecha de publicación del mencionado Reglamento, en evidente contraste con las vías que el mismo texto legal establece para la consecución de los títulos acreditativos para el ejercicio del resto de Especialidades / Disciplinas preventivas (en adelante, "Técnicos o Especialidades Técnicas") reguladas en su Anexo VI y que se han desarrollado -desde entonces- a través de Entidades Formativas Acreditadas por la Autoridad Laboral competente en cada Comunidad Autónoma.

De esta manera, para el caso de las titulaciones sanitarias, concurrieron una serie de condicionantes diferenciales. En primer lugar, se partía ya de la preexistencia de profesionales acreditados para ejercer las funciones previstas, incluso antes de que salieran los nuevos titulados 'técnicos', y así fue como muchos sanitarios asumieron la responsabilidad de los nuevos Servicios de Prevención, que surgían en bastantes casos como "recreados" a partir de los Servicios Médicos de Empresa.

Ahora bien, de cara al futuro, las vías de acceso a los títulos acreditativos 'sanitarios' no sólo no quedaban reguladas en el Reglamento, sino que -en pro de garantizar la calidad dentro del sistema de titulaciones sanitarias- tenía que ser más restrictiva y siguió un curso no ya diferente, sino claramente enlentecido en comparación con las vías abiertas para los "técnicos".

La necesidad de profesionales titulados que siguió al Real Decreto convirtió, de hecho, a los Especialistas de Medicina del Trabajo y a los Diplomados en Enfermería o Medicina de Empresa, hasta entonces poco apreciados como tales, en unos profesionales demandados por la mayoría de empresas que necesitaban y querían organizar sus Servicios de Prevención conforme a norma. Y esta necesidad se acentuó cuando el Consejo Interterritorial del Sistema Nacional de Salud elaboró el informe de Requi- 
sitos para acreditación de las Unidades Básicas Sanitarias integrantes de los Servicios de Prevención acreditados para esta Especialidad.

Pero la oferta (con base en la "producción de nuevos profesionales") no ha respondido -no ha podido responder- a esta demanda. La oferta de titulados sanitarios no ha podido crecer como lo ha hecho entre los profesionales "técnicos", al depender no sólo de las exigencias legislativas del ámbito laboral (competente directo en la materia de riesgos laborales) sino también de una legislación propia: la de ORDENACIÓN DE LAS PROFESIONES SANITARIAS, publicada en 2003, que tiene como objetivo garantizar la competencia profesional de los titulados sanitarios en el conjunto del sistema de salud.

En definitiva, la doble vinculación (dependencia y control) de las autoridades sanitaria y laboral ha lastrado (unas veces ralentizado, y otras dificultado) el acceso de nuevos profesionales a estos títulos y está impidiendo en la práctica que la oferta responda directamente a la demanda del mercado de trabajo.

La oferta de profesionales sanitarios titulados $y / o$ acreditados para este ejercicio ha seguido un proceso no sólo lento (para lo que la demanda exigía) sino también complicado por la necesidad de homologar las diferentes "denominaciones" e incluso de reconocer su competencia a profesionales sin estos títulos pero experimentados y, ejerciendo de hecho, por diferentes razones y sistemas de acceso, las funciones asignadas por el Reglamento de los Servicios de Prevención.

En lo relativo a los médicos, este proceso supone la convivencia de varias titulaciones acreditativas: Médicos de Empresa Diplomados, Médicos Especialistas en Régimen de Alumnado, Médicos Especialistas por reconocimiento de su formación y experiencia (MESTOs) y los Médicos Especialistas en Régimen de Residencia.

Este nuevo procedimiento de formación y acreditación supone un cambio cualititativo en lo concerniente al nivel de exigencia y, por ende de resultados, en las competencias de estos titulados que, a su vez, se está reflejando en una mayor consideración -tanto social como dentro de la profesión médica- de esta Especialidad, lo que responde, por otra parte, a una reclamación histórica de los profesionales que llevamos años dedicados a esta disciplina.

Pero, también, supone un cambio cuantitativo, ya que la acreditación de nuevas Unidades Docentes limita, por sus exigencias, la posibilidad de ampliar la oferta de plazas en las convocatorias MIR y, aún así, desde la primera convocatoria del año 2004 hasta la de este año, la oferta prácticamente se ha triplicado.

Ahora bien, el programa formativo en vigencia corresponde a un periodo de 4 años y por lo tanto, de un año más de lo programado en el curriculum anterior. Ello implica -entre otros efectos- una brecha de un año en el que no se produciría, en buena lógica, el egreso de ningún titulado con la Especialidad de Medicina del Trabajo. Y, a partir de ese cuarto año de formación, una vez completado y estabilizado el proceso, los resultados previsibles son bien limitados: podrán titularse, aproximadamente, 100-150 médicos especialistas por año.

Y todo ello, a pesar del esfuerzo creciente del Ministerio de Sanidad y de las Comunidades Autónomas implicadas (cuyo número, a su vez, se va ampliando año tras año), junto con la inversión de 
algunas Empresas privadas comprometidas también en este empeño y, muy especialmente, del papel de las Mutuas de Accidentes de Trabajo y Enfermedades Profesionales.

En este caso, el Ministerio de Trabajo las ha involucrado muy directamente en el proceso, promoviendo su papel como parte del sistema de formación, pero también, en definitiva, como destino previsible de los profesionales formados. Sin embargo, la coincidencia en el tiempo con los procesos de segregación de las Sociedades de Prevención, en el año 2006, ha supuesto una nueva, otra más, dificultad en el desarrollo del proceso.

En relación con los profesionales de enfermería, el proceso ha sufrido también cambios significativos en los tres últimos años: en 2005 se publicó el Real Decreto de Especialidades que incluye a la de Enfermería del Trabajo entre las nuevas que se proponen y que, por tanto, han de ser reguladas en lo concerniente al proceso formativo (sistema EIR, de enfermeros residentes internos) de varios años y dependientes de Unidades Docentes por acreditar y a los sistemas de reconocimiento y convalidación de las titulaciones preexistentes para lo que se establecen cuatro alternativas, tres de las cuales exigen la superación de una prueba de evaluación cuyas características y convocatoria se establecerán por la Comisión Nacional de la Especialidad, de nueva creación.

Esos tres sistemas permitirán, en su momento, la acreditación de profesionales en alguna de estas situaciones: 4 años de ejercicio; 2 años de ejercicio y 400 horas de formación complementaria en la especialidad. o 1 año de ejercicio y 3 años de enseñanza en áreas de conocimiento relacionados con la especialidad.

Esta Comisión que se ha constituido y puesto en marcha en el año 2007, continúa con sus trabajos y estamos a la espera del informe que realice.

Hasta entonces y, según se recoge en el mencionado Decreto, los cursos que hasta ese año acreditaban para ejercer en los Servicios de Prevención han sido suspendidos, lo que ha producido un retraso de tres años, de momento, en la acreditación de nuevos profesionales.

Por otra parte, y completando el cuadro de oportunidades de formación en esta materia, es obligado incluir las posibilidades de formación continuada de los profesionales que ejercen ya en estos Servicios de Prevención. Este año 2007 se ha publicado el Decreto que regula el sistema de acreditación de la formación continuada, en desarrollo de lo previsto en la Ley 44/2003 y resulta de especial interés en nuestro ámbito las consideraciones recogidas en ella acerca de una concepción de la atención sanitaria integral que "supone la cooperación multidisciplinaria, la integración de los procesos y la continuidad asistencial, y evita el fraccionamiento" reforzando como principio general "La progresiva consideración de la interdisciplinariedad y multidisciplinariedad de los equipos profesionales en la atención sanitaria".

Y, en este sentido, resulta de especial interés recordar la coexistencia en las Unidades de Salud Laboral de los Servicios de Prevención de profesionales con estas titulaciones específicas y otros "con competencia técnica, formación y capacidad acreditada". De tal manera que las necesidades en materia de formación continuada deberán tener en cuenta esta dualidad:

- Para los que ya poseen titulación específica, es de especial interés, concentrar los esfuerzos en la formación para la gestión integrada de la prevención en la empresa y para el trabajo interdisciplinar con la Especialidades Técnicas. 
- Para los sanitarios de los servicios de prevención sin titulación específica se trataría de completar sus perfiles de origen con los temas más específicos de las especialidades en Medicina y Enfermería del Trabajo, seleccionando aquellos temas más característicos y especialmente los relacionados con las actividades preventivas y evaluadoras.

Por último, para ambos, las habilidades en materia de investigación que han sido una carencia reflejada en la realidad de la actividad investigadora de los sanitarios dedicados a la salud laboral si los comparamos con otras especialidades sanitarias.

Al día de hoy, es interés especial de la Escuela Nacional de Medicina del Trabajo investigar para conocer las necesidades en materia de formación continuada de todos los profesionales sanitarios dedicados a la prevención de riesgos laborales y ello, muy especialmente, entre los que realizan su actividad en Unidades de Salud Laboral ya que suelen estar relativamente al margen de los sistemas de formación continuada del Sistema Nacional de Salud. Con este objetivo se propone realizar una investigación específica dirigida a recoger entre los propios profesionales en activo tanto sus inquietudes como sus intereses en este campo, a fin de valorar la pertinencia de programar o promover actividades que las satisfagan.

Ma Josefa Ruiz Figueroa. Médica del Trabajo María Fe Gamo González. Enfermera del Trabajo Escuela Nacional de Medicina del Trabajo. Instituto de Salud Carlos III 